DOI: $10.3901 / J M E .2019 .14 .043$

\title{
LZ50 钢静态再结晶机理及元胞自动机模拟“
}

\author{
师先哲 1,2 杜诗文 ${ }^{1,2}$ \\ (1. 太原科技大学材料科学与工程学院 太原 030024; \\ 2. 金属材料成形理论与技术山西省重点实验室 太原 030024)
}

\begin{abstract}
摘要: LZ50 钢是经典的车轴钢, 但在其锻造过程中组织演变方面的研究存在很多不足。为了探究工艺参数对组织演变的影 响规律, 利用 Gleeble-3500 热模拟试验机对 LZ50 钢进行双道次热压缩模拟试验以分析其静态再结晶行为, 发现高温、高应 变及高应变速率下, 静态再结晶体积分数越大, 温度及应变对静态再结晶体积分数影响也越大, 根据应力应变曲线构建了静 态再结晶动力学模型。元胞自动机模拟结果与试验结果一致, 可以对 LZ50 钢静态再结晶行为进行预测。模拟发现: 温度越 高, 新晶粒生长速度越快; 应变及应变速率越高, 新晶核生成速度越快。电子背散射衍射(EBSD)技术对形变后 LZ50 钢的微 观组织取向分析表明：LZ50 钢静态再结晶的形核方式为晶界弓出形核，在原始晶粒的晶界两侧存在位错密度差，晶界会向 位错密度高的一侧迁移，促进晶核的产生。得到的组织演变规律对生产加工具有指导意义。
\end{abstract}

关键词: LZ50 钢; 静态再结晶; 元胞自动机; 形核机理

中图分类号: TG316

\section{Static Recrystallization Mechanism of LZ50 Steel and Cellular Automata Simulation}

\author{
SHI Xianzhe ${ }^{1,2}$ DU Shiwen ${ }^{1,2}$ \\ (1. School of Materials Science and Engineering, Taiyuan University of Science and Technology, Taiyuan 030024; \\ 2. Shanxi Key Laboratory of Metal Forming Theory and Technology, Taiyuan 030024)
}

\begin{abstract}
Although LZ50 steel is a classic axle steel, there are many deficiencies in the research of microstructure evolution in the forging process. In order to investigate the influence of process parameters on the evolution of microstructure, the static recrystallization behavior of LZ50 steel is analyzed by double-pass hot compression test on Gleeble-3500 thermal simulation machine. It is found that the static recrystallization volume fraction is higher at high temperature, high strain and high strain rate. Temperature and strain have a great influence on the static recrystallization volume fraction. The static recrystallization kinetics model isconstructed according to the stress-strain curve. The simulation results of cellular automata are consistent with the experimental results, which can predict the static recrystallization behavior of LZ50 steel. It can be seen from the simulation that the higher temperature is, the faster new grain growth rate will be, however, the higher strain or strain rateis, the faster the nucleation will be.The electron backscatter diffraction (EBSD) technique is used to analyze the microstructure orientation of LZ50 steel after deformation. The nuclei of static recrystallization forms by bulging of initial grain boundaries. There is a difference in dislocation density onboth sides of original grain boundary. The grain boundary will migrate to the side with high dislocation density to promote the generation of new nuclei. The obtained evolution law of the organization has guiding significance for production and processing.
\end{abstract}

Key words: LZ50 steel; static recrystallization; cellular automaton; nucleation mechanism

\section{0 前言}

近年来，随着高铁的飞速发展，火车轴的需求 量越来越大，并且性能要求越来越高。通常使用快

* 国家自然科学基金资助项目(51305289, 51871158)。20181218 收到初稿, 20190328 收到修改稿。
锻液压机对火车轴进行锻造成形，而锻造过程是间 断性的，在两次锻打期间，材料会根据变形量的多 少发生静态再结晶或亚动态再结晶。当应变量小于 临界应变时, 材料发生静态再结晶, 应变量大于临 界应变则发生亚动态再结晶。而再结晶的发生会降 低材料内部缺陷, 生成细小均匀的晶粒, 提高材料 的强㓞性，使其具有良好的使用性能。再结晶晶粒 
的形核机理是近几年研究中的热点问题, 主要存在 以下几种形核方式: (1) 晶界弓出形核 ${ }^{[1-3]}$ : 形变金 属内晶界两侧会产生位错密度差, 为了降低系统能 量, 晶界会向位错密度高的一侧迁移从而形成晶核。 (2) 亚晶合并形核 ${ }^{[4-5]}$ : 在亚晶结构中, 为了保持界 面张力平衡, 亚晶界发生转动并与周围亚晶合并, 从而产生比周围晶界更大的位向差, 使其可以快速 长大。(3) 孪生形核 ${ }^{[6-7]}$ : 孪晶碰到基体内取向不同 的位错或其它孪晶时, 由于界面相互交叉使得亚晶 转动从而形成大角度晶界, 促进新晶粒形成。(4) 位 错塞积形核 ${ }^{[8-9]}$ : 在第二相析出处或三叉晶界处存在 较高的位错密度也会促进新晶粒的产生。

元胞自动机(Cellular automaton, CA 模型)是 20 世纪 50 年代左右由 Von Neumann 提出来模拟生物 机体自我繁殖演变的一种模型 ${ }^{[10]}$, 近 20 年来已经 被运用到材料领域并在模拟材料形变过程中的组织 演变方面扮演重要角色。相比于蒙特卡洛法与相场 法, 元胞自动机法在模拟过程中可以得到每一时刻 位错密度、晶粒尺寸等定量信息, 并且考虑了变形 对晶界的影响, 引入曲率驱动机制, 模拟过程更加 接近实际物理现象 ${ }^{[11-12]}$ 。国外, 伦敦大学的 DING 等 ${ }^{[13]}$ 认为再结晶是连接介观位错活动与宏观力学 性能的关键, 他们结合冶金原理与元胞自动机, 综 合考虑温度及应变速率的影响建立了再结晶模型, 并且该模型可以精准的预测材料在再结晶过程中的 微观组织演变。JANSSENS ${ }^{[14]}$ 则解释了 CA 模型中 具体参数的物理意义及模型计算的具体过程, 并提 出了一种关于 CA 模型的先进概念, 即不规则形状 的元胞自动机。国内, ZHENG 等 ${ }^{[15]}$ 运用 CA 模型 研究了多道次轧制中材料的再结晶行为, 并且模拟 结果与试验结果具有良好的一致性。XIAO 等 ${ }^{[16]}$ 将 $\mathrm{CA}$ 模拟与基于矢量运算的拓扑形变技术进行耦合, 利用该技术模拟微观组织演变时, 发现再结晶速度 更快, 并且得到的再结晶晶粒更加细小。CHEN 等 ${ }^{[17]}$ 通过建立描述晶粒变形的物质坐标系与描述晶粒形 核和长大的元胞坐标系, 对 $30 \mathrm{Cr} 2 \mathrm{Ni} 4 \mathrm{MoV}$ 不同应变 速率及温度下的动态再结晶进行模拟。他们在模拟 过程中均采用晶粒拓扑技术使模拟结果与试验结果 更加接近。LIN 等 ${ }^{[18]}$ 等利用 CA 模型对镍基超合金进 行研究时考虑了晶界处溶质元素对晶界迁移的影 响, 并且研究了道次间隔时间对静态再结晶中晶粒 均匀性的影响及再结晶过程中晶粒尺寸的变化。

尽管人们在再结晶机理及组织演变模拟等方面 做了很多工作, 但目前 LZ50 车轴钢的研究仅限于 其瘦劳性能或使用寿命, 因此本文以 LZ50 钢为研 究对象, 揭示其静态再结晶机理并且运用 CA 模型
模拟 LZ50 钢的组织演变。

\section{1 试验内容}

试验用 LZ50 钢的化学成分(质量分数)如表 1 所 示, 将试验试样切成 $\phi 10 \mathrm{~mm} \times 15 \mathrm{~mm}$ 的圆柱并在 Gleeble-3500 热模拟试验机上进行压缩试验。在试 样与砧子之间加入钽片及高温润滑剂以减少摩擦对 应力状态的影响, 并增加导电性。试验具体流程如 图 1 所示。

表 1 LZ50 钢的化学成分 $\%$

\begin{tabular}{ccccc}
\hline $\mathrm{C}$ & $\mathrm{Mn}$ & $\mathrm{Si}$ & $\mathrm{P}$ & $\mathrm{S}$ \\
$0.47 \sim 0.57$ & $0.60 \sim 0.90$ & $0.17 \sim 0.40$ & $\leqslant 0.03$ & $\leqslant 0.03$ \\
\hline $\mathrm{Cr}$ & $\mathrm{Ni}$ & $\mathrm{Cu}$ & $\mathrm{Al}_{\mathrm{s}}$ & $\mathrm{Fe}$ \\
$\leqslant 0.30$ & $\leqslant 0.30$ & $\leqslant 0.25$ & $\geqslant 0.01$ & 其余 \\
\hline
\end{tabular}

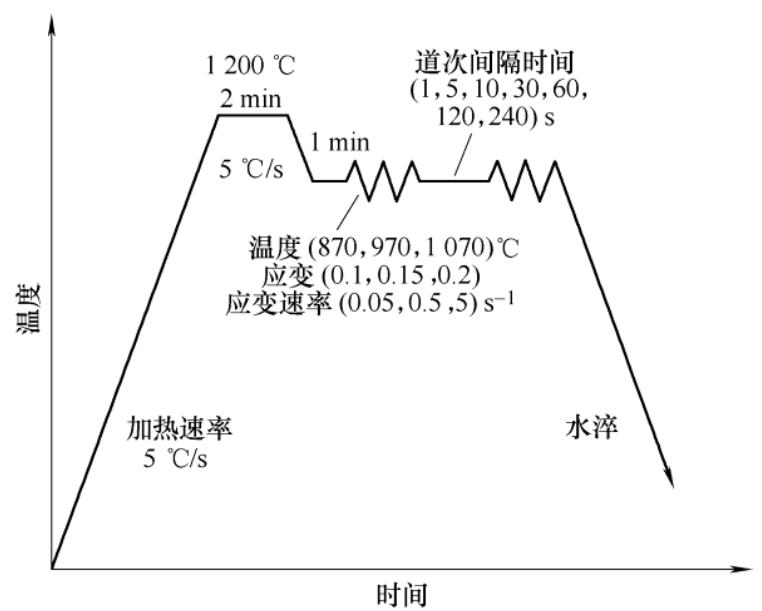

图 1 双道次热压缩试验流程图

考虑温度的影响: 将试样以 $5{ }^{\circ} \mathrm{C} / \mathrm{s}$ 的速度加热 至 $1200{ }^{\circ} \mathrm{C}$, 保温 $2 \mathrm{~min}$ 后以 $5{ }^{\circ} \mathrm{C} / \mathrm{s}$ 的速度降至 $870{ }^{\circ} \mathrm{C} 、 970{ }^{\circ} \mathrm{C}$ 及 $1070{ }^{\circ} \mathrm{C}$ 并保温 $1 \mathrm{~min}$ 以消除材 料内部温度梯度, 第一、二道次真应变均为 0.15 , 压缩速率为 $0.5 \mathrm{~s}^{-1}$, 道次间保温时间为: 压缩温度 为 $870{ }^{\circ} \mathrm{C}$ 时保温 $10 \mathrm{~s} 、 30 \mathrm{~s} 、 60 \mathrm{~s} 、 120 \mathrm{~s} 、 240 \mathrm{~s}$; 压缩温度为 $970{ }^{\circ} \mathrm{C}$ 时保温 $5 \mathrm{~s} 、 10 \mathrm{~s} 、 30 \mathrm{~s} 、 60 \mathrm{~s} 、 120$ $\mathrm{s}$; 压缩温度为 $1070{ }^{\circ} \mathrm{C}$ 时保温 $1 \mathrm{~s} 、 5 \mathrm{~s} 、 10 \mathrm{~s} 、 30 \mathrm{~s}$ 、 $60 \mathrm{~s}$, 试验结束后水冷。

考虑变形量的影响: 将试样以 $5{ }^{\circ} \mathrm{C} / \mathrm{s}$ 的速度加 热至 $1200{ }^{\circ} \mathrm{C}$, 保温 $2 \mathrm{~min}$ 后以 $5{ }^{\circ} \mathrm{C} / \mathrm{s}$ 的速度降至 $970{ }^{\circ} \mathrm{C}$ 并保温 $1 \mathrm{~min}$, 第一道次真应变分别为 0.1 、 0.2 , 压缩速率为 $0.5 \mathrm{~s}^{-1}$, 第二道次真应变与第一道 次相同，道次间保温时间为 $5 \mathrm{~s} 、 10 \mathrm{~s} 、 30 \mathrm{~s} 、 60 \mathrm{~s}$ 、 $120 \mathrm{~s}$, 试验结束后水冷。

考虑变形速率的影响: 将试样以 $5{ }^{\circ} \mathrm{C} / \mathrm{s}$ 的速度 加热至 $1200{ }^{\circ} \mathrm{C}$, 保温 $2 \mathrm{~min}$ 后以 $5{ }^{\circ} \mathrm{C} / \mathrm{s}$ 的速度降 至 $970{ }^{\circ} \mathrm{C}$ 并保温 $1 \mathrm{~min}$, 第一、二道次真应变均为 
0.15 , 压缩速率为 $0.05 \mathrm{~s}^{-1} 、 5 \mathrm{~s}^{-1}$, 道次间保温时间 为 $5 \mathrm{~s} 、 10 \mathrm{~s} 、 30 \mathrm{~s} 、 60 \mathrm{~s} 、 120 \mathrm{~s}$, 试验结束后水冷。

考虑原始组织的影响: 将试样以 $5{ }^{\circ} \mathrm{C} / \mathrm{s}$ 的速度 加热至 $1020{ }^{\circ} \mathrm{C} 、 1070{ }^{\circ} \mathrm{C} 、 1120{ }^{\circ} \mathrm{C}$, 保温 $2 \mathrm{~min}$ 后以 $5{ }^{\circ} \mathrm{C} / \mathrm{s}$ 的速度降至 $970{ }^{\circ} \mathrm{C}$ 并保温 $1 \mathrm{~min}$, 第一、 二道次真应变均为 0.15 , 压缩速率为 $0.5 \mathrm{~s}^{-1}$, 道次 间保温时间为 $5 \mathrm{~s} 、 10 \mathrm{~s} 、 30 \mathrm{~s} 、 60 \mathrm{~s} 、 12 \mathrm{ss}$, 试验结 束后水冷。

试验结束后将试样沿压缩方向切成两半, 一半 镶嵌进行腐蚀试验以观察晶粒尺寸, 腐蚀液为 $85{ }^{\circ} \mathrm{C}$ 过饱和苦味酸溶液加少量十二烷基苯磺酸钠 和盐酸腐蚀 $90 \mathrm{~s}$; 另一半进行电解抛光以分析晶粒 织构取向及静态再结晶机理, 电解液为 $10 \%$ 高氯酸 加 $90 \%$ 乙醇, 电解电压为 $20 \mathrm{~V}$, 时间为 $120 \mathrm{~s}$, 温 度为零下 $20{ }^{\circ} \mathrm{C}$ 。

\section{2 试验结果与分析}

材料热成形过程中由于金属流动导致内部位错 堆积, 从而发生加工硬化; 但同时位错的局部堆积 又积攒了大量能量, 这些能量成为了静态再结晶的 驱动力, 再结晶会使材料产生软化行为。通常计算 软化率的方法主要有应力补偿法、后插法、5\%总应 变法及平均应力法 ${ }^{[19-20]}$ 。为了减少人为处理数据的 误差, 采取应力补偿法计算软化率

$$
\varphi=\frac{\sigma_{\mathrm{m}}-\sigma_{2}}{\sigma_{\mathrm{m}}-\sigma_{1}}
$$

如图 2 所示, $\sigma_{1} 、 \sigma_{2}$ 分别为第一、二道次压 缩时的屈服应力; $\sigma_{\mathrm{m}}$ 为第一道次结束时的应力。为 了消除回复对静态再结晶体积分数计算的影响, 认 为当软化率超过 0.2 时才会发生静态再结晶, 因此 软化率与静态再结晶体积分数 $X_{\mathrm{srx}}$ 之间的关系为 ${ }^{[21]}$

$$
X_{\mathrm{srx}}=(\varphi-0.2) /(1-0.2)
$$

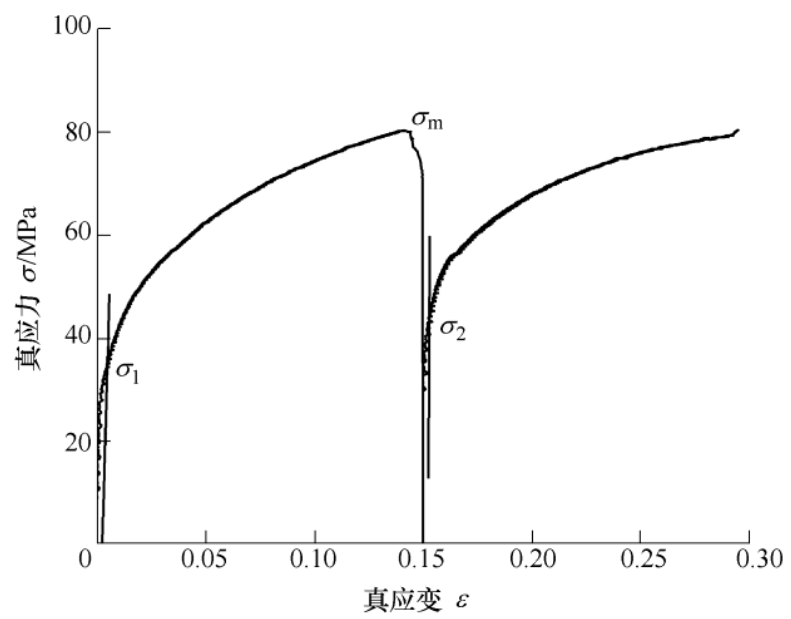

图 2 软化率计算示意图

\section{1 工艺参数对静态再结晶体积分数的影响}

在热成形过程中静态再结晶体积分数 $X_{\mathrm{srx}}$ 会受 到温度、应变、应变速率及原始组织的影响。如图 3 所示为各条件下道次间隔时间 $t$ 与 $X_{\mathrm{srx}}$ 的关系图。 通常, 随着保温时间的增加, 静态再结晶体积分数 $X_{\mathrm{s} r \mathrm{~s}}$ 会不断增大，但其增长速率越来越慢。

由图 $3 \mathrm{a}$ 可以看出在应变及应变速率不变的情 况下, 变形温度为 $1070{ }^{\circ} \mathrm{C}$ 时, 静态再结晶体积分 数更大并且增长的速度最快, 仅在 $60 \mathrm{~s}$ 就达到了 $78.4 \%$ 。温度为 $970{ }^{\circ} \mathrm{C}$, 保温时间为 $60 \mathrm{~s}$ 时, $X_{\text {srx }}$ 为 $45.2 \%$ 。相同条件下, 温度为 $870{ }^{\circ} \mathrm{C}$ 时, $X_{\mathrm{srx}}$ 为 $29.4 \%$, 增长速率最慢。这主要是由于温度越高, 静态再结晶的储能越多 ${ }^{[22]}$, 同时温度越高, 晶界迁 移速率越快, 使得再结晶晶核形成及长大速率越快。

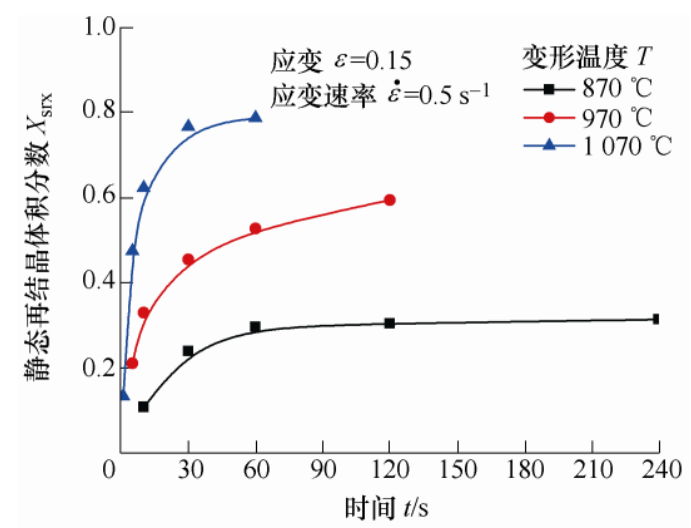

(a) 变形温度

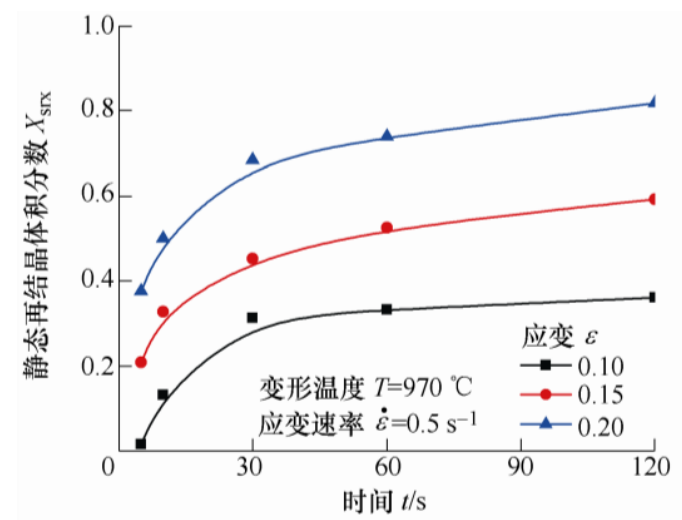

(b) 应变

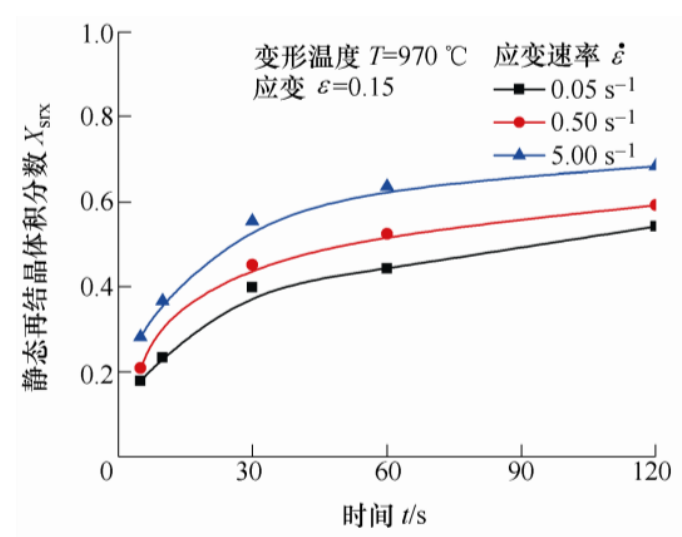

(c) 应变速率 


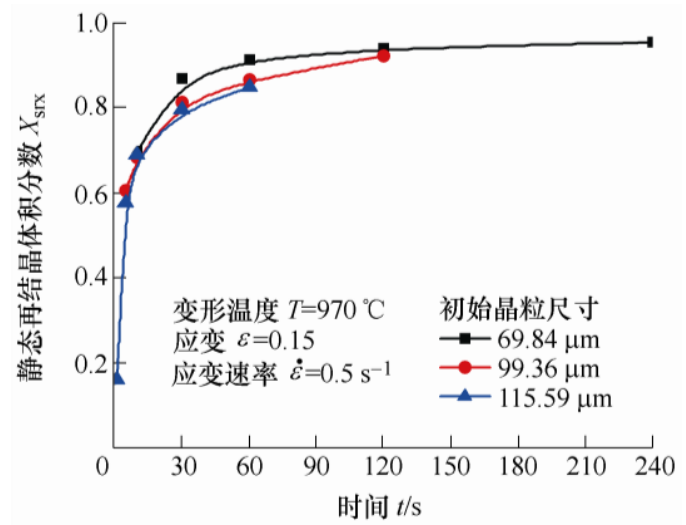

(d) 初始晶粒尺寸

图 3 各工艺参数对静态再结晶体积分数的影响

图 $3 \mathrm{~b}$ 中各曲线温度及应变速率保持一致, 当真 应变为 0.2 , 道次间隔时间为 $120 \mathrm{~s}$ 时 $X_{\mathrm{srx}}$ 为 $81.7 \%$; 真应变为 0.1 时 $X_{\mathrm{srx}}$ 等于 $36.1 \%$, 可以看出, 真应变 差 0.1 时, 静态再结晶体积分数相差了 $45.6 \%$ 。这 是因为真应变为 0.1 时, 金属流动小, 位错累计较 少; 而真应变为 0.2 已经非常接近 LZ50 钢的临界应 变, 材料内部已经有进行动态再结晶的趋势, 说明 此时材料内位错密度很高, 应变储能非常大。为了 降低系统体积自由能，材料将在道次间隔时间内发 生静态再结晶。

图 $3 \mathrm{c}$ 比较了相同温度及应变时, 不同应变速 率对 $X_{\mathrm{srx}}$ 的影响。可以看出, 道次间隔时间为 120 $\mathrm{s}$ 时, 应变速率为 $0.05 \mathrm{~s}^{-1}$ 及 $5 \mathrm{~s}^{-1}$ 的 $X_{\mathrm{srx}}$ 相差 $15.6 \%$ 。这是由于应变速率越高, 变形时间越短, 材料发生回复的时间越短, 从而消耗的位错就越 少; 并且应变速率越大, 材料加工硬化率越大, 位错累积速度越快。因此, 材料应变速率越大, 位错密度越大, 再结晶驱动力越大, 静态再结晶 进行的越充分。

图 3d 对比了不同初始晶粒尺寸对 $X_{\mathrm{srx}}$ 的影响, 可以看出道次间隔时间相同时, 不同晶粒尺寸下 $X_{\mathrm{srx}}$ 相差很小, 说明初始晶粒尺寸对静态再结晶体 积分数的影响不大。

可以看出, 温度及应变对静态再结晶体积分数 影响较大, 其次为应变速率, 而初始晶粒尺寸对静 态再结晶体积分数的影响最小。根据静态再结晶体 积分数与时间的关系可以做出静态再结晶速率图 (图 4), 不难看出, 道次间隔时间为 $5 \mathrm{~s}$, 压缩温度 分别为 $970{ }^{\circ} \mathrm{C}$ 及 $1070{ }^{\circ} \mathrm{C}$ 时, 静态再结晶速率相差 $0.034 \mathrm{~s}^{-1}$; 对应变及应变速率进行分析发现, 最大 静态再结晶速率未超过 $0.013 \mathrm{~s}^{-1}$ 。说明温度越高, 静态再结晶进行的速率越快, 而不同应变及应变速 率下，静态再结晶速率相差较小。

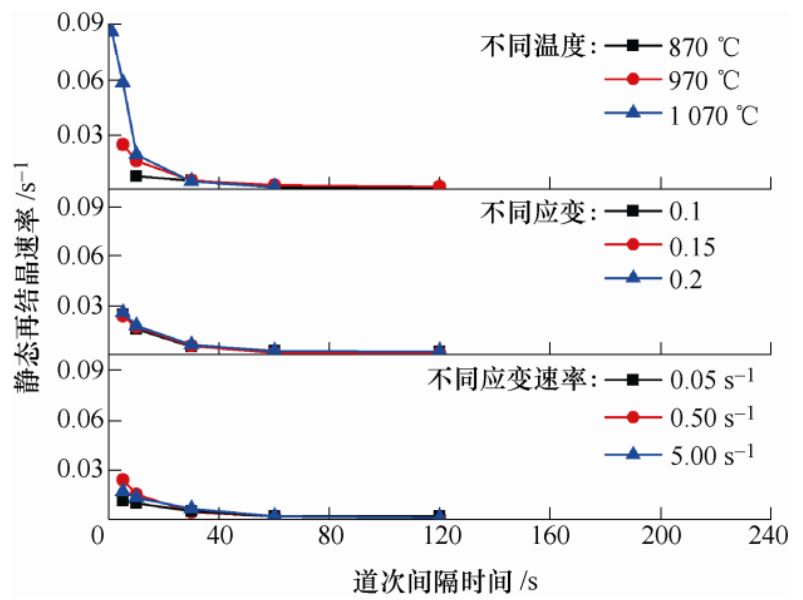

图 4 静态再结晶速率

\section{2 静态再结晶动力学模型}

静态再结晶的动力学方程可以用 Avrami 方程 ${ }^{[23]}$ 表示

$$
X_{\text {srx }}=1-\exp \left[-0.693\left(\frac{t}{t_{0.5}}\right)^{k}\right]
$$

式中, $k$ 为材料常量, $t$ 为道次间隔时间, $t_{0.5}$ 为静 态再结晶发生 $50 \%$ 的时间。 $t_{0.5}$ 可用下式表示

$$
t_{0.5}=a d_{0}{ }^{h} \varepsilon^{m} \dot{\varepsilon}^{n} \exp \left(\frac{Q_{\text {srx }}}{R T}\right)
$$

式中, $d_{0}$ 为原始晶粒尺寸, $\varepsilon$ 为应变, $\dot{\varepsilon}$ 为应变速 率, $Q_{\mathrm{srx}}$ 为静态再结晶激活能, $T$ 为热力学温度, $R$ 为气体常数, $a 、 h 、 m$ 和 $n$ 为材料相关系数。通 过线性拟合可以得到 LZ50 钢的静态再结晶动力学 方程

$$
\left\{\begin{array}{l}
X_{\text {srx }}=1-\exp \left[-0.693\left(\frac{t}{t_{0.5}}\right)^{0.421}\right] \\
t_{0.5}=2.1 \times 10^{-10} d_{0}^{0.018} \varepsilon^{-4.805} \dot{\varepsilon}^{-0.287} \exp \left[\frac{281088}{R T}\right]
\end{array}\right.
$$

\section{3 静态再结晶 $\mathrm{CA}$ 模拟}

第一道次压缩结束后的保温时间内材料发生静 态再结晶。模拟假设道次间隔时间内温度不变, 各 位置形核率、位错密度一致。本文主要模拟 LZ50 钢压缩后静态再结晶行为, 首先通过拓扑变形技术 对模型进行压缩处理。在二维模型中, 任意一点都 可以通过向量进行表示, 设 $\boldsymbol{a}$ 为原始矢量, $\boldsymbol{b}$ 为变 形后的新矢量, $S$ 为变形矩阵, 变形过程可由下式 表示 ${ }^{[16]}$

$$
b=S a
$$

矩阵的表示方式为

$$
\left(\begin{array}{l}
\boldsymbol{b}_{x} \\
\boldsymbol{b}_{y}
\end{array}\right)=\left(\begin{array}{cc}
l_{x} & 0 \\
0 & l_{y}
\end{array}\right)\left(\begin{array}{l}
\boldsymbol{a}_{x} \\
\boldsymbol{a}_{y}
\end{array}\right)
$$


$\boldsymbol{a}_{x} 、 \boldsymbol{a}_{y}$ 分别为原始矢量, $\boldsymbol{b}_{x} 、 \boldsymbol{b}_{y}$ 为新矢量, $l_{x} 、 l_{y}$ 的数值与真应变相关。

新晶粒的形成包括形核与长大两个过程, 模拟 中平均形核率可以由 IVASISHIN 等 ${ }^{[24]}$ 所使用的形 核率模型确定

$$
\dot{n}(t)=C\left[H(t)-H_{\text {min }}\right] V_{\Omega}(t) \exp \left(-\frac{Q_{\text {srx }}}{R T}\right)
$$

式中, $C$ 为形核参数; $V_{\Omega}(t)$ 表示 $t$ 时刻材料可以继 续生成再结晶晶核的概率; $H(t)$ 为 $t$ 时刻的储存 能; $H_{\text {min }}$ 为材料发生再结晶所需的最小储存能, 能发 生再结晶的最小变形量通常在 $2 \% \sim 10 \%$ 范围内 ${ }^{[25]}$, 因此 $H_{\text {min }}$ 为材料发生 $2 \% \sim 10 \%$ 形变时对应的储存 能。 $H$ 可以通过式(9)计算

$$
H=C_{0} \rho \mu b^{2} V_{\mathrm{r}}
$$

式中, $C_{0}$ 为常数, 通常取 $0.5 \sim 1.0 ; \mu$ 为剪切模量; $b$ 为伯格斯矢量; $V_{\mathrm{r}}$ 为奥氏体摩尔体积, 为 7.2 $\mathrm{cm}^{2} / \mathrm{mol} ; \rho$ 为位错密度。

再结晶晶粒长大的速度可由式(10)表示 ${ }^{[26]}$

$$
v=M F /\left(4 \pi r^{2}\right)
$$

式中, $M$ 为晶界迁移率, $F$ 为晶粒长大的驱动力。 晶界迁移率 $M$ 可由式(11)计算

$$
M=\frac{\delta D_{\mathrm{ob}} b}{K T} \exp \left(-\frac{Q_{b}}{R T}\right)
$$

式中, $\delta$ 为特定材料的晶界厚度; $D_{\mathrm{ob}}$ 为晶界自扩 散系数; $K$ 为波尔兹曼常数, $K=1.3806505 \times 10^{-23}$ $\mathrm{J} / \mathrm{K} ; Q_{b}$ 为晶界自扩散激活能。

再结晶晶粒长大的驱动力

$$
F=4 \pi r^{2}(\tau \Delta \rho-2 \gamma / r)
$$

式中, $\tau$ 为单位长度位错线能; $\gamma$ 为界面能, 可由 式(13) (15)计算

$$
\begin{gathered}
\tau=0.5 \mu b^{2} \\
\gamma= \begin{cases}\gamma_{\mathrm{m}} & \theta \geqslant 15^{\circ} \\
\gamma_{\mathrm{m}} \frac{\theta}{\theta_{\mathrm{m}}\left(1-\ln \frac{\theta}{\theta_{\mathrm{m}}}\right)} & \theta<15^{\circ}\end{cases} \\
\gamma_{\mathrm{m}}=\frac{\mu b \theta_{\mathrm{m}}}{4 \pi(1-v)}
\end{gathered}
$$

式中, $\theta$ 为两晶粒的取向差, 通常认为取向差大于 $15^{\circ}$ 为大角度晶界, 小于 $15^{\circ}$ 为小角度晶界; $\gamma_{\mathrm{m}}$ 为大 角度晶界能; $v$ 为泊松比。

图 5 为试样加热至 $1200{ }^{\circ} \mathrm{C}$, 保温 $2 \mathrm{~min}$ 后的 金相组织图, 平均晶粒尺寸为 $168.83 \mu \mathrm{m}$; 图 $6 \mathrm{a}$ 为 $1200{ }^{\circ} \mathrm{C}$ 下通过晶粒长大模拟得到的原始组织, 图 $6 \mathrm{~b}$ 为真应变为 0.15 的原始组织图, 平均晶粒尺

寸为 $169.63 \mu \mathrm{m}$ 。变形后, 在面积不变的前提下, 晶界所占比例却要高于变形前, 并且沿压缩方向的 再结晶晶粒碰撞距离也会变短, 这样最终形核位点 增加，再结晶晶粒形状大小也会发生改变。

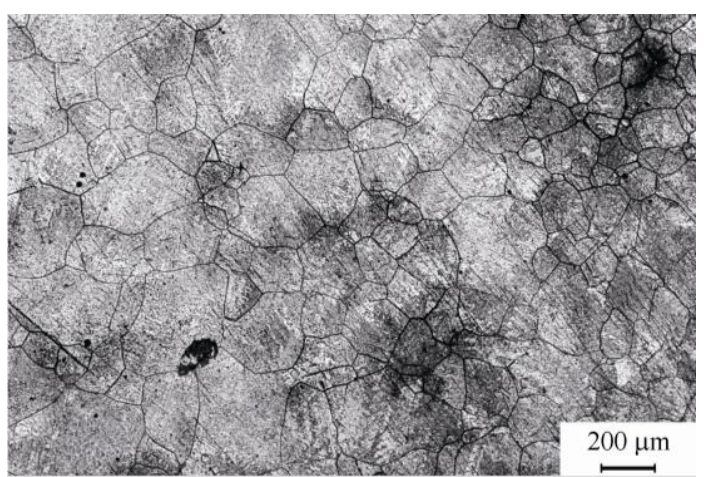

图 $51200{ }^{\circ} \mathrm{C}$ 保温 $2 \mathrm{~min}$ 的金相组织

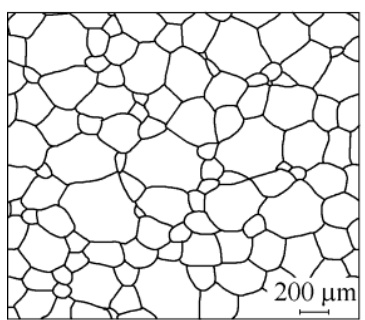

(a) 真应变为 0

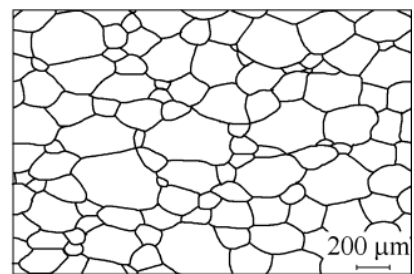

(b) 真应变为 0.15

图 6 原始组织

图 7 为金相图与模拟结果的对比。970-0.5-0.150.15-30 表示加工时变形温度为 $970{ }^{\circ} \mathrm{C}$, 应变速率 为 $0.5 \mathrm{~s}^{-1}$, 双道次真应变均为 0.15 , 道次间隔时间 为 $30 \mathrm{~s}$ 。其中, 彩色部分表示再结晶晶粒, 不同颜 色代表不同的晶粒取向。可以看出，相同条件下温 度越高, 应变越大, 应变速率越快, 组织内部能量 越多, 组织演变更容易发生。由图 8、9 可以看出, 不同条件下模拟得到的晶粒尺寸及静态再结晶体积 分数与试验结果保持很好的一致性, 说明 $\mathrm{CA}$ 模型 可以很好地应用于组织演变的研究。

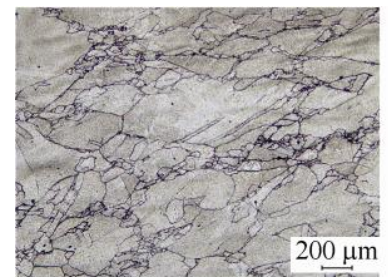

(a)

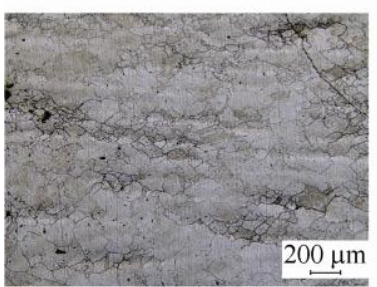

(c)

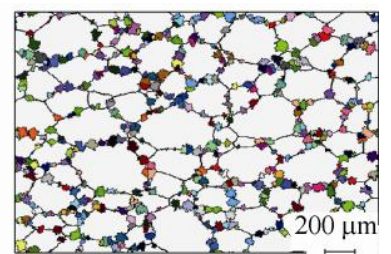

(b)

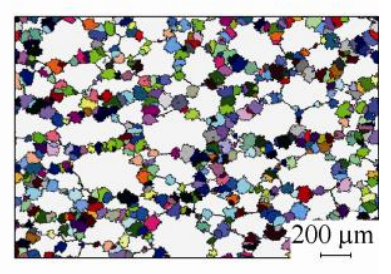

(d) 


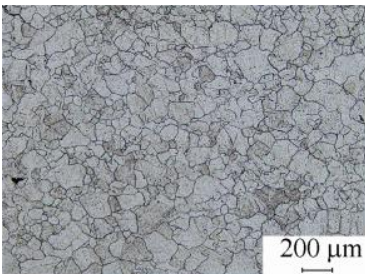

(e)

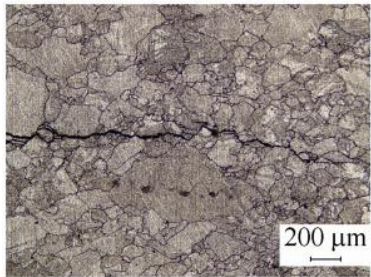

(g)

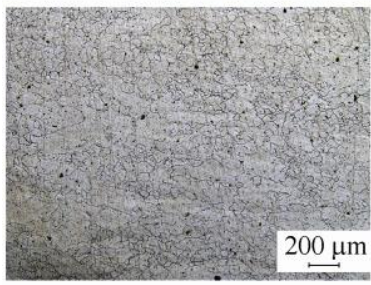

(i)

(k)

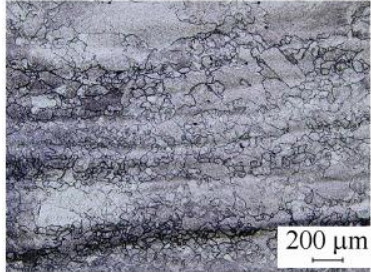

(m)

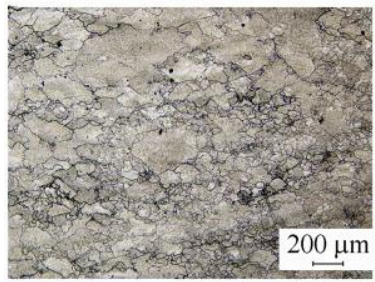

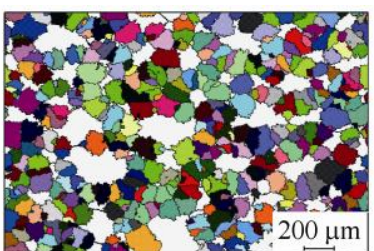

(f)

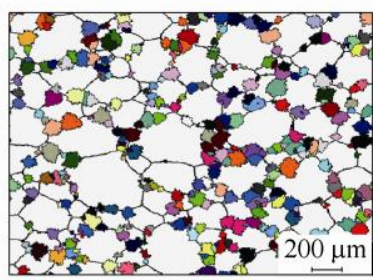

(h)

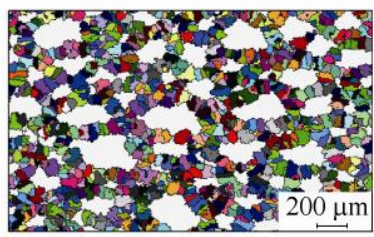

(j)

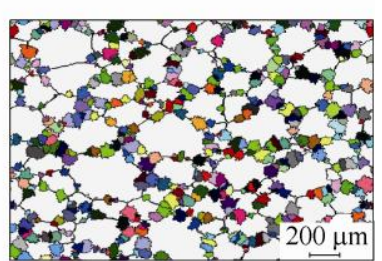

(1)

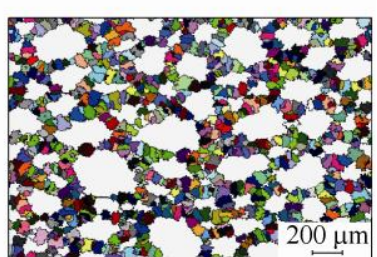

(n)
图 7 静态再结晶金相组织与模拟对比图

(a)(b), (c)(d), (e)(f): 870, 970, 1070-0.5-0.15-0.15-30;

(g)(h): 970-0.5-0.1-0.1-30;(i)(j): 970-0.5-0.2-0.2-30;

$(\mathrm{k})(1),(\mathrm{m})(\mathrm{n}): 970-0.05,0.5-0.15-0.15-30$

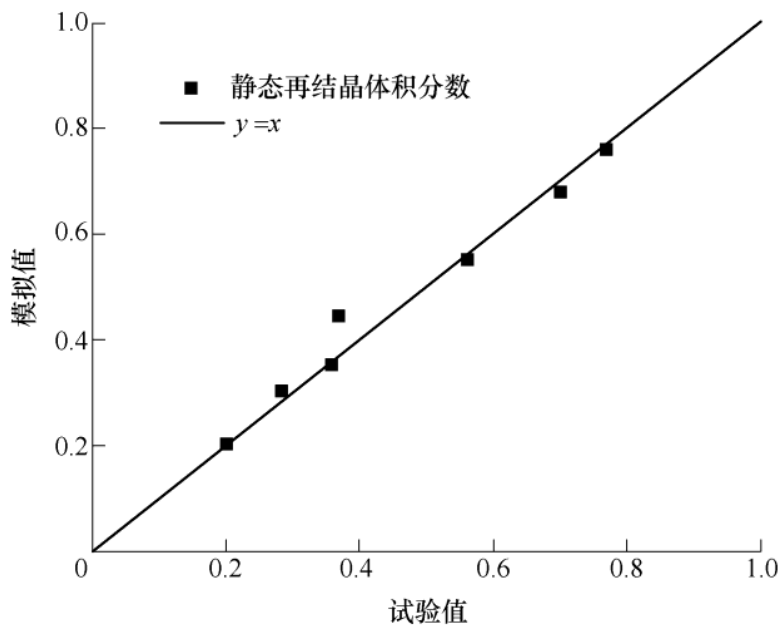

图 8 静态再结晶体积分数试验值与模拟值对比

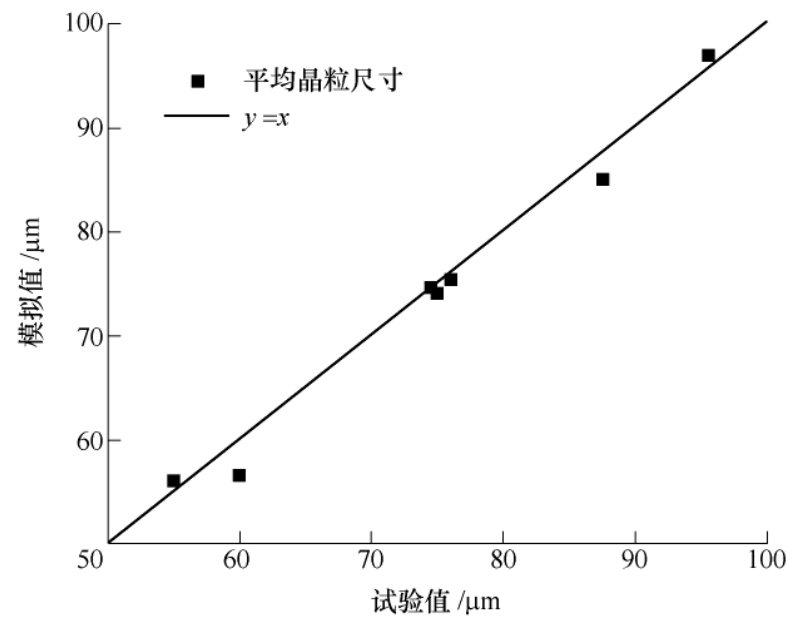

图 9 平均晶粒尺寸试验值与模拟值对比

图 10 为利用 $\mathrm{CA}$ 模型模拟的不同温度、应变 及应变速率下静态再结晶晶粒尺寸演变规律。温 度越高, 平均晶粒尺寸越大, 而应变及应变速率 越高, 新晶粒数量越多。这是由于变形温度为 $1070{ }^{\circ} \mathrm{C}$ 时, 此时温度较高, 为原子扩散提供了大 量能量, 晶界迁移速率加快, 晶粒长大速度变快, 再结晶晶核可以快速成长为大晶粒。当应变量较 大时, 热加工结束后材料内部储存能增加, 再结 晶驱动力越大, 形核率增加, 晶粒更加细小。应 变速率越高，材料在热加工期间回复效果越弱, 加工硬化引起位错密度大大增加, 从而为再结晶 的发生提供了有利条件。再结晶晶粒的平均直径 可由下式表达

$$
d=K_{1}\left(\frac{G}{\dot{N}}\right)^{1 / 4}
$$

式中, $K_{1}$ 为材料常数, $G$ 为长大线速度, $\dot{N}$ 为形 核率。晶粒大小取决于 $G / \dot{N}$, 温度较高时, 晶界 迁移速度更快，应变及应变速率较大时，位错密 度更大，储存能高。显然，高应变及高应变速率 下 $G / \dot{N}$ 的数值较高温条件下要低, 因此晶粒尺寸 更小。

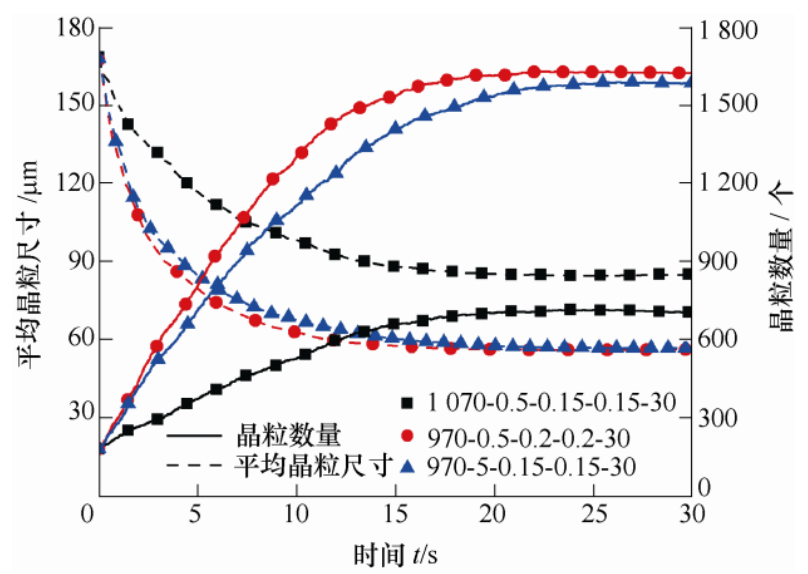

图 10 不同工艺参数下平均晶粒尺寸与晶粒数量 


\section{4 静态再结晶机理}

图 11 为 LZ50 钢初始组织, 可以看出未变形之前, 组织极其不均匀, 晶粒粗大且形状不规则, 晶粒尺寸 达到了 $443.23 \mu \mathrm{m}$ 。经过热加工, 材料内部位错密度 迅速升高从而为再结晶提供驱动力, 随后经保温孕 育, 产生许多细小均匀的再结晶晶粒(图 12)。静态再 结晶初期, 材料内体积自由能较高, 晶界迁移及亚晶 长大会消耗位错以降低体积自由能, 因此小角度晶界 比例较高。而通过延长道次间隔时间, 升高变形温度, 提高加工速率, 再结晶晶粒形核长大速度加快, 再结 晶体积分数增加, 大量新晶粒出现, 此时小角度晶界 减少, 而大角度晶界增加, 如图 13 , 小于 $15^{\circ}$ 为小角 度晶界, 大于 $15^{\circ}$ 小于 $60^{\circ}$ 为大角度晶界。

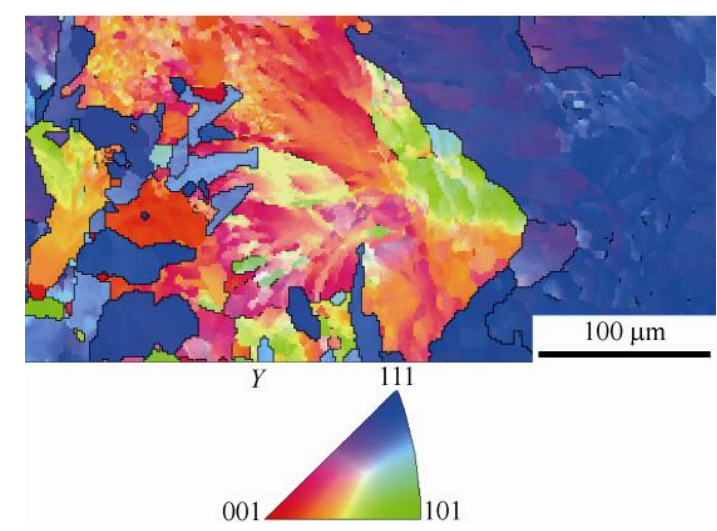

图 11 原始组织晶粒取向图

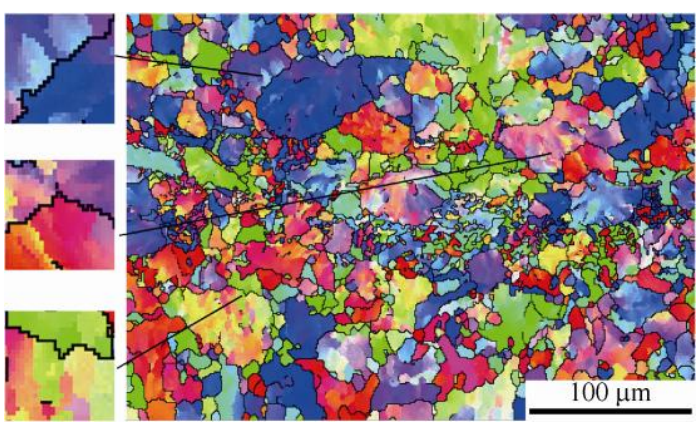

图 12 970-0.5-0.15-0.15-10 晶粒取向图

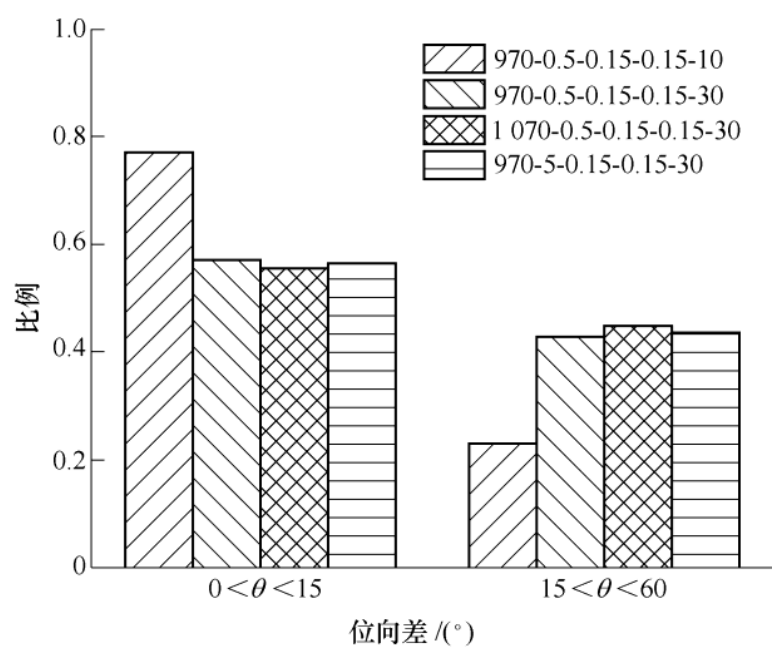

图 13 大小角度晶界比例图
图 14 为高倍数下变形温度为 $970{ }^{\circ} \mathrm{C}$, 真应变为 0.15 , 应变速率为 $0.5 \mathrm{~s}^{-1}$, 道次间隔时间为 $10 \mathrm{~s}$ 的 大角度晶界图。在原始晶粒内分别取平行于晶界及 垂直于晶界的两条线分析其累计取向差及与起始点 相关的取向差。累计取向差为点与点之间的取向差, 与起始点相关的取向差为每一个点与第一个点的取 向差。如图 15 所示, 由 $A_{1}$ 可以看出, 平行于原始

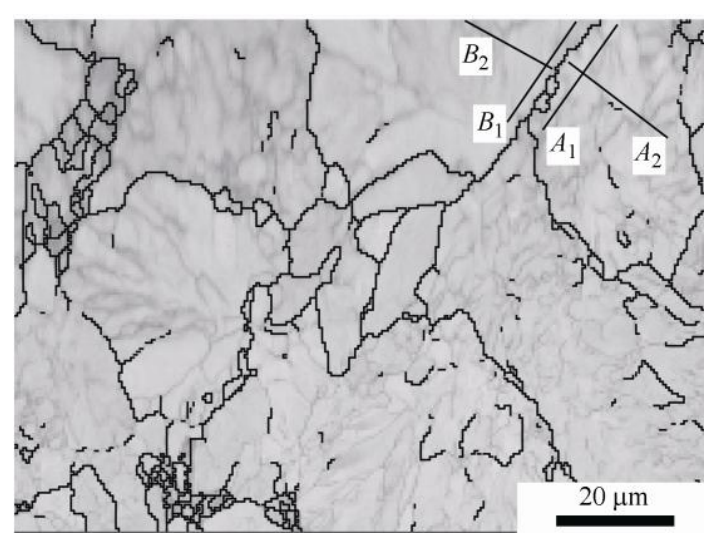

图 14 大角度晶界图

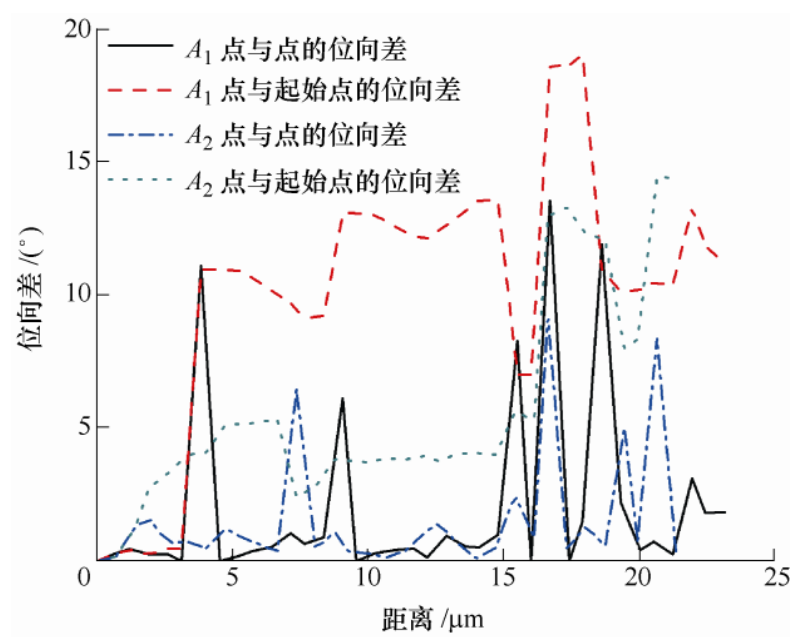

(a)

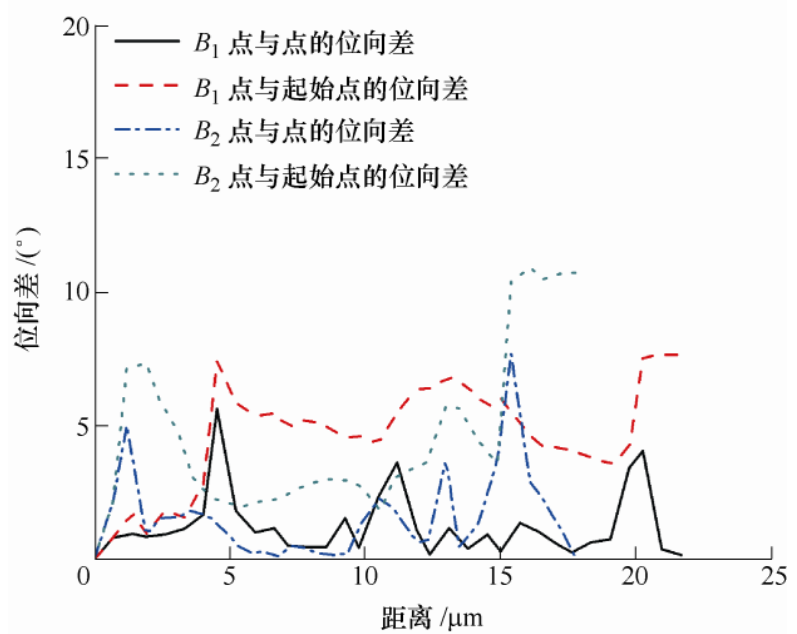

(b)

图 15 原始晶粒内位向差变化图 
晶界处, 存在 5 个大于 $5^{\circ}$ 的位向差极大值, 最大的 位向差达到了 $13.5^{\circ}$, 与第一点的位向差最高为 $18.9^{\circ}$; 在垂直于原始晶界的方向 (线段 $\mathrm{A}_{2}$ ), 点与点 的最大位向差为 $9.2^{\circ}$, 晶粒中心与晶界附近位向差 最大为 $14.4^{\circ}$; 说明在晶界附近位向差的变化大于晶 粒心部。研究表明, 位向差的大小可以反映位错密 度的高低, 反之亦然 ${ }^{[27]}$ 。因此, 该晶粒在晶界附近 会发生较大的变形，使得位错在晶界处聚集。

在相邻晶粒内做同样的线段, 发现在晶界附近 (线段 $B_{1}$ ), 只有一点与周围取向差超过了 $5^{\circ}$, 而第 一点与其余点的位向差最大到达了 $7.6^{\circ}$; 在垂直于 晶界的方向(线段 $B_{2}$ ) 发现累计取向差最大为 $7.7^{\circ}$, 第一点与其余点的取向差最大为 $10.9^{\circ}$; 垂直晶界方 向位向差的变化大于晶界附近, 而该晶粒晶界附近 位向差较小说明此处受到的变形较小, 因此只有少 量位错在此处聚集。

WUSATOWSKA-SARNEK 等 ${ }^{[28]}$ 在对铜的研究 中发现新晶粒在锯齿形晶界处产生, 并且适当角度 的亚晶及孪晶也会激发再结晶的进行。PAGGI 等 ${ }^{[29]}$ 在对奥氏体不锈钢的研究中发现形核发生在静态再 结晶的初期, 而应变诱导晶界迁移在接下来的晶粒 长大过程中扮演重要角色。可以看出在图 14 中大角 度晶界上有新晶粒产生, 在未发生再结晶的大角度 晶界处也可以观察到锯齿形的晶界 (图 12)。这是由 于压缩过程中, 首先材料内部应变不均匀, 其次回 复过程中相邻晶粒内结构调整方式不同均导致了大 角度晶界附近的位错密度不同, 这种差异会驱动晶 界朝着位错密度高的一方弓弯, 即形成锯齿形晶界。 锯齿形晶界的出现可以降低体积自由能, 消耗大量 位错, 使整个系统趋于稳定, 且这种晶界将进一步 促进晶核的产生。

\section{3 结论}

(1) 温度越高, 应变越大, LZ50 钢静态再结 晶进行的更充分, 而应变速率对静态再结晶的影 响次之, 初始晶粒尺寸的影响最小。温度对静态 再结晶速率影响最大; 不同应变及应变速率下, 静态再结晶速率相差不大。构建了静态再结晶动 力学方程。

(2) 模拟结果与试验结果一致, 温度越高, 再 结晶晶粒长大速度更快, 而应变及应变速率越高, 静态再结晶的形核速度更快。选择合适的变形温 度, 即 $970{ }^{\circ} \mathrm{C}$ 左右, 保证质量的前提下选择更高 的应变速率 (大于等于 $0.5 \mathrm{~s}^{-1}$ ) 可以获得细小均匀 的晶粒。
(3) 形变后 LZ50 钢原始晶界两侧的位错密 度存在差异，晶界向位错密度高的一侧迁移以促 进形核, LZ50 钢静态再结晶形核方式为晶界弓 出形核。

\section{参 考 文 献}

[1] AZARBARMAS M, AGHAIE-KHAFRI M, CABRERA $\mathrm{J}$ M, et al. Dynamic recrystallization mechanisms and twining evolution during hot deformation of Inconel 718[J]. Materials Science and Engineering A , 2016, 678: 137-152.

[2] BABU K A, MANDAL S, ATHREYA C N, et al. Hot deformation characteristics and processing map of a phosphorous modified super austenitic stainless steel[J] Materials and Design, 2017, 115: 262-275.

[3] 韦康, 张麦仓, 谢锡善. 超超临界电站用镍基合金热加 工过程的再结晶机理 [J]. 金属学报, 2017，53(12): 1611-1619.

WEI Kang , ZHANG Maicang, XIE Xishan. Recrystallization mechanisms in hot working processes of a nickel-based alloy for ultra-supercritical power plant application[J]. ACTA Metallurgica Sinica, 2017, 53(12): 1611-1619.

[4] KIMS I, YOOYC. Continuous dynamic recrystallization of AISI 430 ferritic stainless steel[J]. Metals and Materials, 2002, 8(1): 7-13.

[5] 万菊林, 孙新军, 顾家琳, 等. Al-Cu-Mg-Zn-Cr 合金热 扭转变形中连续动态再结晶机理 $[\mathrm{J}]$. 金属学报, 1999, 35(10): 1031-1035.

WAN Julin, SUN Xinjun, GU Jialin, et al. Research on continuous dynamic recrystallization in hot torsion of Al-Cu-Mg-Zn-Cr alloy[J]. ACTA Metallurgica Sinica, 1999, 35(10): 1031-1035.

[6] WON J W, LEE T, HONG S G, et al. Role of deformation twins in static recrystallization kinetics of high-purity alpha titanium[J]. Metals and Materials International, 2016, 22(6): 1041-1048.

[7] 间辰㑆, 曲寿江, 冯艾寒, 等. 钛及钛合金形变孪晶的 研究进展[J]. 稀有金属，1999，35(10)：1031-1035. YAN Chenkan, QU Shoujiang, FENG Aihan, et al. Recent advances of deformation twins in titanium and titanium alloys[J]. Chinese Journal of Rare Metals，1999，35(10): 1031-1035.

[8] MIURA H, ANDIARWANTO S, SATO $\mathrm{K}$, et al Preferential dynamic nucleation at triple junction in copper tricrystal during high-temperature deformation[J]. Materials Transactions, 2002, 43(3): 494-500. 
[9] 钟茜婷, 王否, 刘峰. Incoloy 028 合金不连续动态再结 晶中链状组织形成机理研究 [J]. 金属学报, 2018, 54(7): 969-980.

ZHONG Qianting, WANG Lei, LIU Feng. Study on formation mechanism of necklace structure in discontinuous dynamic recrystallization of Incoloy 028[J]. ACTA Metallurgica Sinica， 2018， 54(7): 969-980.

[10] 陈飞, 崔振山, 董定乾. 微观组织演变元胞自动机模拟 研究进展[J]. 机械工程学报, 2015，51(4): 30-39.

CHEN Fei, CUI Zhenshan, DONG Dingqian. Research progress in cellular automaton simulation of microstructure evolution[J]. Journal of Mechanical Engineering, 2015, 51(4): 30-39.

[11] 陈飞. 热锻非连续变形过程微观组织演变的元胞自动 机模拟[D]. 上海: 上海交通大学, 2012.

CHEN Fei. Simulation of microstructureevolution during discontinuous hot forging processes using cellular automaton method[D]. Shanghai: Shanghai Jiao Tong University, 2012.

[12] 郭一娜. 金属环件铸辗复合成形过程微观组织演变的 元胞自动机模拟[D]. 太原: 太原科技大学，2014.

GUO Yina. Microstructureevolution simulation during metal ring blank-casting and rolling compound forming processes using cellular automaton method[D]. Taiyuan:

Taiyuan University of Science and Technology, 2014.

[13] DING R, GUO Z X. Microstructural modelling of dynamic recrystallisation using an extended cellular automaton approach[J]. Computational Materials Science, 2002, 23(1-4): 209-218.

[14] JANSSENS K G F. An introductory review of cellular automata modeling of moving grain boundaries in polycrystalline materials[J]. Mathematics and Computers in Simulation, 2010, 80(7): 1361-1381.

[15] ZHENG Chengwu, XIAO Namin, LI Dianzhong, et al. Microstructure prediction of the austenite recrystallization during multi-pass steel strip hot rolling: A cellular automaton modeling[J]. Computational Materials Science, 2008, 44(2): 507-514.

[16] XIAO Namin, ZHENG Chengwu, LI Dianzhong, et al. A simulation of dynamic recrystallization by coupling a cellular automaton method with a topology deformation technique[J]. Computational Materials Science, 2008, 41(3): 366-374.

[17] CHEN Fei, CUI Zhenshan, LIU Juan, et al. Mesoscale simulation of the high-temperature austenitizing and dynamic recrystallization by coupling a cellular automaton with a topology deformation technique[J]. Materials Science and Engineering A, 2010, 527(21):
5539-5549.

[18] LIN Yongcheng, LIU Yanxiang, CHEN Mingsong, et al. Study of static recrystallization behavior in hot deformed Ni-based superalloy using cellular automaton model[J]. Materials and Design, 2016, 99: 107-114.

[19] RAO K P, PRASAD Y K D, HAWBOLT E B. Study of fractional softening in multi-stage hot deformation[J]. Journal of Materials Processing Technology, 1998, 77(1-3): 166-174.

[20] FERNÁNDEZ A I, LÓPEZ B, RODRÍGUEZ-IBABE J M. Relationship between the austenite recrystallized fraction and the softening measured from the interrupted torsion test technique[J]. Scripta Materialia, 1999, 40(5): 543-549.

[21] SUN W P, HAWBOLT E B. Comparison between static and metadynamic recrystallization - An application to the hot rolling of steels[J]. ISIJ International, 1997，37(10): 1000-1009.

[22] 萄永诚, 陈明松, 钟掘. $42 \mathrm{CrMo}$ 钢形变奥氏体的静态 再结晶[J]. 中南大学学报, 2009, 40(2): 411-416.

LIN Yongcheng, CHEN Mingsong, ZHONG Jue. Static recrystallization behaviors of deformed $42 \mathrm{CrMo}$ steel[J] Journal of Central South University, 2009, 40(2) : 411-416.

[23] 贾璐, 李永堂, 李振晓. 耐热合金钢 P91 热变形过程静 态及亚动态再结晶行为 [J]. 机械工程学报, 2017,53(8): 58-67.

JIA Lu , LI Yongtang, LI Zhenxiao. Static and metadynamic recrystallization behaviors of Heat-resistant P91 alloy during hot deformation[J]. Journal of Mechanical Engineering, 2017， 53(8): 58-67.

[24] IVASISHIN O M , SHEVCHENKO S V , VASILIEV N L , et al. A 3-D Monte-Carlo (Potts) model for recrystallization and grain growth in polycrystalline materials[J]. Materials Science and Engineering A, 2006, 433(1-2): 216-232.

[25] 崔忠圻, 覃耀春. 金属学与热处理 $[\mathrm{M}]$. 北京: 机械工 业出版社, 2007.

CUI Zhongqi, TAN Yaochun. Metallography and heat treatment $[\mathrm{M}]$. Beijing: China Machine Press, 2007.

[26] 陈双梅. LZ50 钢火车轴快锻成形过程的动态再结晶行 为[D]. 太原：太原科技大学，2016.

CHEN Shuangmei. Dynamic recrystallization behavior of LZ50 steel train shaft in rapid forging process[D]. Taiyuan: Taiyuan University of Science and Technology, 2016.

[27] 孟杨, 任群, 鞠新华. 利用局域取向差衡量变形金属中 
的位错密度 $[\mathrm{J}]$. 材料热处理学报, 2014, 35(11): 122-128.

MENG Yang, REN Qun, JU Xinhua. Evaluation of dislocation density by local grain misorientation in deformed metals $[\mathrm{J}]$. Transactions of Materials and Heat Treatment, 2014, 35(11): 122-128.

[28] WUSATOWSKA-SARNEK A M, MIURA H, SAKAI T. Nucleation and microtexture development under dynamic recrystallization of copper[J]. Materials Science and Engineering A, 2002, 323(1-2): 177-186.
[29] PAGGi A, ANGELla G, DONNINI R. Strain induced grain boundary migration effects on grain growth of an austenitic stainless steel during static and metadynamic recrystallization[J]. Materials Characterization, 2015, 107: 174-181.

作者简介: 师先哲, 男, 1994 年出生。主要研究方向为材料加工先进制 造技术。

E-mail: 864466453@qq.com

杜诗文(通信作者), 女, 1975 年出生, 博士，教授，硕士研究生导师。 主要研究方向为材料加工先进制造技术。

E-mail: tykddsw@126.com 